\title{
Identifying and measuring the behavioural, dietary, and physical activity components of weight management consultations delivered by general practice nurses in routine care
}

\author{
Heather Tong ${ }^{1}$, Elizabeth Morris ${ }^{1}$, Susan A. Jebb ${ }^{1,2}$ and Dimitrios A. Koutoukidis ${ }^{1,2^{*}}$ (D)
}

\begin{abstract}
Background: Many people with obesity receive weight loss consultations by general practice nurses (GPNs) in routine primary care. This exploratory study aimed to characterise the components of these consultations, including behaviour change techniques (BCTs), and dietary and physical activity recommendations.

Methods: We analysed audio recordings of weight management consultations conducted by 8 GPNs as part of the 'usual care' group in a randomised controlled trial (ISRCTN75092026). Consultations were coded against three taxonomies to classify BCTs, dietary recommendations, and physical activity recommendations. Associations between coded content and weight loss were assessed. Differences in the content of consultations where weight loss was $<5 \%$ or $\geq 5 \%$ from baseline weight at 6 months were explored.

Results: One hundred and fifty audio recordings were available from 53 out of 140 (38\%) participants in the usual care group. Participants had on average $3(S D=1)$ recorded consultations over 3 months, lasting $14(S D=7)$ minutes each. Weight change at 3, 6, and 12 months was $-3.6 \%(S D=4.3),-5.5 \%(S D=6.0)$ and $-4.2 \%(S D=6.5)$ for participants with audio recordings. GPNs used $3.9(\mathrm{SD}=1.6)$ of $93 \mathrm{BCTs}, 3.3(\mathrm{SD}=2.7)$ of 30 dietary recommendations and $1.4(\mathrm{SD}=1.2)$ of 10 physical activity recommendations per consultation. The most commonly employed BCTs were feedback on outcome of behaviour (80.0\%), problem solving (38.0\%), and social reward (34.3\%). The most common dietary recommendations were about portion size (31.3\%), nutrients (28.0\%), and balanced diet (19.7\%). The main physical activity recommendation was about walking (30.3\%). There was no association between weight loss and the number of dietary recommendations, physical activity recommendations, or BCTs used per consultation, or per participant. Social reward was the only technique used significantly more in consultations of participants that lost $\geq 5 \%$ of their baseline weight at 6 months.
\end{abstract}

Conclusions: The study provides a new method that could be used to describe the content of weight management consultations. Specific dietary or physical activity recommendations and BCTs were used infrequently and inconsistently in this group of GPNs. Although replication is required in larger samples, this may point to a weakness in current practice.

Keywords: Diet, Physical activity, Behaviour change technique, Primary care, Weight loss

\footnotetext{
${ }^{*}$ Correspondence: dimitrios.koutoukidis@phc.ox.ac.uk

${ }^{1}$ Nuffield Department of Primary Care Health Sciences, University,

of Oxford, Radcliffe Observatory Quarter, Woodstock Road, Oxford OX2

$6 \mathrm{GG}, \mathrm{UK}$

Full list of author information is available at the end of the article
}

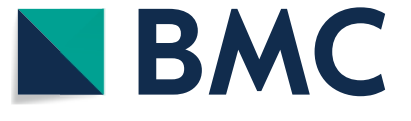

(c) The Author(s) 2021. Open Access This article is licensed under a Creative Commons Attribution 4.0 International License, which permits use, sharing, adaptation, distribution and reproduction in any medium or format, as long as you give appropriate credit to the original author(s) and the source, provide a link to the Creative Commons licence, and indicate if changes were made. The images or other third party material in this article are included in the article's Creative Commons licence, unless indicated otherwise in a credit line to the material. If material is not included in the article's Creative Commons licence and your intended use is not permitted by statutory regulation or exceeds the permitted use, you will need to obtain permission directly from the copyright holder. To view a copy of this licence, visit http://creativecommons.org/licenses/by/4.0/. The Creative Commons Public Domain Dedication waiver (http://creativeco mmons.org/publicdomain/zero/1.0/) applies to the data made available in this article, unless otherwise stated in a credit line to the data. 


\section{Contributions to the literature}

- This study provides a method to analyse the content of weight management consultations.

- The use of recognised behaviour change techniques or dietary and physical activity recommendations by GPNs was infrequent and variable.

- Results should be generalised with caution, because these GPNs reported training in weight management and self-identified as confident in this area of care

\section{Background}

Obesity affects over $25 \%$ of the UK's population and is a major preventable risk factor for morbidity and mortality [1]. In addition to affecting individuals, obesity places considerable financial strain on healthcare systems and the wider society [2].

Weight loss achieved through changes in diet and physical activity has many benefits including reducing the risk of developing type 2 diabetes, significantly reducing levels of cardiovascular risk factors, such as hypertension, and is associated with a reduction in premature mortality [3-5]. Therefore, weight loss interventions for people with obesity are an important part of wider strategies to reduce non-communicable diseases.

The National Institute for Health and Care Excellence (NICE) recommends that primary care professionals offer behavioural weight management support to people with obesity [6]. One-to-one weight management consultations with a practice GPN are common in the primary care setting. Randomised control trials (RCTs) also frequently use 'usual care' interventions as a comparator setting, although the content of these sessions is often neither standardised nor recorded. It is recommended by NICE that weight management consultations should involve discussion of behavioural, dietary, and physical activity change, aiming to achieve a $600 \mathrm{kcal}$ daily energy deficit [6]. This is expected to lead to a $0.5-1 \mathrm{~kg}$ weight loss per week [7]. Despite this, most literature finds primary care weight management consultations, including those delivered by practice GPNs, lead to minimal average weight loss, but with substantial variation $[8,9]$.

Improvements in outcomes may be facilitated by improvements in intervention delivery, but this requires an initial understanding of how GPNs typically conduct weight management consultations [10]. One method of characterising the content of weight management consultations is through the use of taxonomies. Taxonomies enable reporting of complex interventions by identifying 'active ingredients': replicable and irreducible components that make an intervention effective. They also establish a common language and can be helpful in translating effective interventions into practice [10]. Characterising the behavioural, diet and physical activity components of GPN-led weight management consultations may allow for the active ingredients of more effective routine consultations to be identified and facilitate intervention optimisation.

This study aimed to address the evidence gap in the content of nurse-led weight management consultations by characterising the behavioural, diet and physical activity components in a small group of consultations. In addition, based on evidence that greater use of behaviour change techniques (BCTs) leads to greater weight loss [11], we aimed to identify whether more intensive use of techniques and recommendations would be associated with weight loss in this study.

\section{Methods}

This was a secondary analysis of data from the Doctor Referral of Overweight People to Low Energy total diet replacement Treatment (DROPLET) trial. The DROPLET trial recruited 278 participants seeking to lose weight and with a $\mathrm{BMI} \geq 30 \mathrm{~kg} / \mathrm{m}^{2}$. Participants were then randomly allocated to a total diet replacement programme or usual care. One hundred and forty participants were allocated to the usual care group, and of this group, 53 participants had their consultations audio-recorded. We report on data from all 53 individuals in the usual care group that had their consultations recorded to avoid introducing further selection bias.

Usual care comprised consultations with a GPN for the explicit purpose of weight loss [12]. The GPNs conducting the consultations had self-identified as confident in delivering a weight management programme. They were asked to continue consultations in their usual manner but additionally received the British Heart Foundation booklet 'So you want to lose weight for good' to use at their discretion. Consultations took place in general practices in the UK. Consultations from the trial were audio-recorded, and participants' weights were recorded at baseline, 3, 6, and 12 months [12].

\section{Audio recordings}

Participants provided written informed consent for their consultations to be recorded. The recordings were not anonymised but were stored securely. MP3 audio recordings of weight management consultations were classified as 'introductory' or 'follow-up'. 'Introductory' sessions were the initial meeting between the GPN and each participant, whilst 'follow-ups' were subsequent consultations intended to monitor and support the participants. Two audio recordings could not be linked to individual 
participants, so they were included in descriptive coding but not the analysis of weight change.

\section{Taxonomy development, use, and coding}

Weight management consultations given by GPNs were coded against three components: $\mathrm{BCTs}$ using the $\mathrm{BCT}$ taxonomy version 1 (BCTTv1) [13], dietary recommendations using a modified version of the Stok 2018 diet taxonomy [14], and physical activity recommendations using an investigator-designed physical activity taxonomy.

The BCTTv1 was chosen as it is an extensive consensually used taxonomy, allowing the identification of BCTs in weight management consultations. The NICE behaviour change guidelines use this taxonomy and include examples of how to use BCTs in the context of weight loss [15]. BCTTv1 contains 93 BCTs grouped into a hierarchy of 16 domains, with examples of a realistic use of each [13]. Three researchers (HT, EM, DAK) coded the content using the established BCTTv1 definitions, available in additional file 1, and examples following completion of a standardised online training for BCTTv1.

The Stok 2018 diet taxonomy was the only diet-specific taxonomy identified within the literature [14]. After listening to randomly selected sample data, the taxonomy was revised to capture the recommendations in the context of weight management consultations more thoroughly. Five recommendations were added: 'ability to pay', 'rate of eating,' 'food and drink substitution', 'total energy intake', and 'alcoholic drink intake'. The recommendation 'dieting' was removed, so that the specific recommendations used to encourage dieting, a purpose of the consultations, could be quantified. The adapted taxonomy had 30 recommendations within the domains of 'food choice', 'eating behaviour', or 'dietary intake and nutrition. The dietary taxonomy used is available in additional file 2 . Researchers coded the content against the adapted taxonomy with pre-set definitions and as examples were not present in the original taxonomy, these were discussed and developed in a code rulebook, available in additional file 3.

A pre-existing physical activity taxonomy could not be identified in the literature, so we developed a taxonomy of 10 recommendations based on the FITT principle (frequency, intensity, time, and type) and a review of existing physical activity interventions [16]. The FITT principle was chosen because it highlights the 4 main planning stages of physical activity. Domains 'lifestyle activity' and 'structured activity' were chosen as Public Health England (PHE) recognises these as forms of physical activity [17]. The third domain with the single category 'sedentary time' was included because sedentary time is independently associated with mortality and morbidity and new
PHE guidelines recommend reductions in sedentary time $[17,18]$. The physical activity taxonomy used is shown in additional file 4 .

Techniques/recommendations were rated as absent (coded as 0 ), present in all probability (coded as 1 ), or present beyond all reasonable doubt (coded as 2), as per the BCT training guidance [19]. Coding BCTs/ recommendations as 1 or 2 was based on the confidence of presence, as it was apparent BCTs/ recommendations met the criteria of definitions to differing degrees. One instance of the category present was sufficient to code the content. Consultations were listened to a minimum of 2 times. Coders were blinded to participants' baseline weight and overall weight change until all coding was complete.

One researcher coded all consultations. Two further researchers independently coded a random sample (10\%) of the consultations against the three taxonomies. Coded in batches of two or three, researchers then met to discuss the coding. Justification of the rationale for coding specific $\mathrm{BCTs} /$ recommendations was discussed. Techniques/ recommendations that any researcher coded in consultations that were not coded by both other researchers were discussed in detail. This process was repeated iteratively until an agreement between all 3 coders was reached and final codes were applied.

A code rulebook was developed to aid coding. This consisted of rules on when to code specific BCTs/ recommendations and examples that should and shouldn't be coded. For example, the BCT social reward was coded as ' 1 ' if the GPN gave general reward (e.g. a GPN said well done to a participant, not directly referring to one behaviour in particular but generally from the overall outcome at the end of consultation). It was coded as ' 2 ' if the GPN gave reward about a specific behaviour or outcome of behaviour (e.g. if the GPN congratulated the participant for losing $1 \mathrm{~kg}$ after weighing them). General coding rules stated to only code what the GPN said. It was not sufficient to code a $\mathrm{BCT}$ or recommendation if a GPN was simply responding to a participant's statement, unless the GPN developed a discussion about the suggestion for an extended period of time. Guidance also stated that when GPNs questioned a participant about their behaviours, it was not sufficient to code questions asking about the past (e.g. 'Have you swum before?'), but it was sufficient to code if the GPN asked questions relating to the future (e.g. 'Will you try swimming next week?').

\section{Inter-rater reliability}

Three random consultations coded for BCTs and 3 for dietary and physical activity recommendations were 
independently coded by each researcher, following iterative discussion and independent coding in triplicate of $10 \%$ of the consultations. Perfect agreement was considered when all coders coded the same values $(0,1$ or 2) for a technique/ recommendation. Good and poor agreement, calculated using Krippendorff's alpha with a SPSS syntax by Hayes, was considered to be an alpha value $\geq 0.600$ and $<0.600$, respectively.

\section{Data Analysis}

Analyses were carried out in Microsoft Excel v15 and SPSS v25.0 (Chicago, IL). Continuous variables are presented as means and standard deviations. Independent t-tests compared weight change from baseline at 3, 6, and 12 months in audio-recorded and non-audio-recorded groups. The Mann-Whitney $\mathrm{U}$ test was used to compare lengths of introductory and follow-up consultations.

Percentage usage of BCTs and recommendations across audio-recorded consultations was calculated. A weighted mean of each $\mathrm{BCT}$ and recommendation used in consultations was calculated. BCTs and recommendations coded as 1 were weighted half of those coded as 2 , allowing for quantification of the fact that some BCTs were coded as present with more confidence than others.

For the following tests, BCTs and recommendations rated as 1 or 2 were deemed present and calculations were based on presence or absence rather than confidence of presence. Associations between lengths of consultations, and $\mathrm{BCT}$ and recommendation use with weight loss were assessed with the Pearson's correlation coefficient.

Comparisons of the average and total length of consultations as well as the average number of BCTs and recommendations used per consultation were made between participants that had lost at least or less than $5 \%$ of their baseline weight at 6 months. This was based on the transtheoretical model of intentional behaviour change which argues long-term changes in behaviour may be assessed after 6 months [20]. Percentage usage of individual BCTs and recommendations used in consultations of the subgroup was compared using chi-squared tests and where appropriate Fisher's exact test (Table S3, additional file 5).
Due to small numbers, we plotted the weight change and the usage of techniques/ recommendations per consultation by GPN, but were unable to perform additional formal exploratory analysis (Additional file 5, figures S1-4).

The level of statistical significance was set at $\mathrm{p}<0.05$.

\section{Results}

\section{Data characteristics}

Audio recordings were available for 53 out of 140 participants (38\%) in the usual care group of the trial from consultations at 7 practices in Oxfordshire by 8 GPNs. Three additional GPNs delivering consultations did not return any recordings. Participants' characteristics are detailed in Table 1. Twenty-eight out of 150 audio-recorded consultations (19\%) were classified as 'introductory' and 122 out of 150 (81\%) as 'follow-up'. Participants in the audio-recorded group had on average 2.8 (SD 1.2) consultations over 3 months, between 1 to 6 in total. The average consultation length per participant was 13.8 (SD 6.7) minutes, ranging from 3.1 to $21.7 \mathrm{~min}$, with no difference in duration between introductory and follow-up consultations $(p=0.14)$. The average total length of all recorded consultations per participant was 36.9 (SD 20.8; range 9.3-82.4) minutes. The British Heart Foundation booklet was discussed in some introductory consultations (20.4\%), where it was briefly summarised and given to participants as an extra aide to look through in their own time. It was only discussed rarely thereafter in $4.9 \%$ of follow-up consultations.

\section{Weight change at 3,6, and 12 months}

Weight change in participants with audio-recorded consultations at 3, 6, and 12 months was $-3.6 \%$ (SD 4.3), $-5.5 \%$ (SD 6.0), and $-4.2 \%$ (SD 6.5), respectively. In those without audio recordings, weight change at 3, 6, and 12 months was $-3.0 \%$ (SD 3.4), $-3.8 \%$ (SD 5.4), and $-2.4 \%$ (SD 6.4), respectively. In absolute terms, participants with audio-recorded consultations lost $3.5 \mathrm{~kg}$ (SD 4.4), $5.5 \mathrm{~kg}$ (SD 6.3), and $4.0 \mathrm{~kg}$ (SD 6.7) at 3, 6, and 12 months, respectively, and participants without audio recordings lost $3.1 \mathrm{~kg}$ (SD 4.0), $3.8 \mathrm{~kg}$ (SD 6.1), and $2.4 \mathrm{~kg}$ (SD 6.4), respectively. This modest weight change did not differ

Table 1 Demographic characteristics of participants

\begin{tabular}{llll}
\hline Characteristic & $\begin{array}{l}\text { All usual care participants } \\
(\boldsymbol{n}=\mathbf{1 4 0 )}\end{array}$ & $\begin{array}{l}\text { Participants with recordings } \\
(\boldsymbol{n}=\mathbf{5 1})\end{array}$ & $\begin{array}{l}\text { Participants without recordings } \\
(\boldsymbol{n}=\mathbf{8 9})\end{array}$ \\
\hline Age (years) & $47.6(12.7)$ & $49.7(13.7)$ & $46.2(11.9)$ \\
Sex, female (\%) & 60.7 & 64.7 & 58.4 \\
Ethnicity, white (\%) & 86.4 & 82.4 & 88.7 \\
Baseline weight (kg) & $105.1(19.9)$ & $101.2(17.7)$ & $107.3(20.8)$ \\
\hline
\end{tabular}

Data presented as mean (SD) or percentage 
significantly between participants with or without audio recordings at 3 months $(p=0.37), 6$ months $(p=0.09)$, or 12 months $(p=0.13)$.

\section{Inter-rater reliability}

Of the $93 \mathrm{BCTs}, 80$ were coded with perfect agreement between all coders. A further 2 BCTs had good levels of agreement and 11 BCTs had poor agreement. For dietary recommendations, 25,1 , and 4 recommendations and, for physical activity, 8,1 , and 1 recommendations were coded with perfect, good, and poor agreement, respectively.

\section{Characterising BCTs, dietary recommendations, and physical activity recommendations used in consultations}

Of 93 possible BCTs, 29 were coded at least once in any consultation from 14 out of 16 domains. Of 30 possible dietary recommendations, 24 were used from all 3 domains and 9 out of 10 physical activity recommendations were used from 2 out of 3 domains (Table 2). The three most frequently used BCTs were 'feedback on outcome of behaviour' (used in $80.0 \%$ of consultations), 'problem solving' (38.0\%) and 'social reward' (34.3\%). The top three dietary recommendations were 'portion size' (31.3\%), 'nutrients' (28.0\%), and 'diversity of pattern' (19.7\%). The most frequent physical activity recommendation was encouragement of walking (30.3\%) (Fig. 1).

\section{Associations between consultations, components and outcomes}

The length of consultations was significantly positively associated with the number of BCTs present $(r=0.45$, $p=0.01)$. The length of consultation was not associated with the number of dietary $(r=0.28, p=0.16)$ or physical activity recommendations $(r=0.04, p=0.83)$. The total length of all audio recordings a participant received was not associated with weight change at $3(r=-0.03, p=0.84)$, $6(r=0.01, p=0.93)$, or $12(r=-0.05, p=0.78)$ months.

There were no significant associations between the total number of BCTs, dietary or physical activity recommendations used in participants' introductory consultations with weight change at 3,6 , or 12 months or the total number used across all of a participant's consultations and weight change at 3, 6 or 12 months (Table 3). There were also no significant correlations between the average numbers of BCTs, dietary or physical activity recommendations used per consultation with weight change at 3,6 , or 12 months, with the exception of an association between the average number of physical activity recommendations used per consultation and weight gain at 12 months only $(r=0.36(p=0.02)$, Tables S1-S3, additional file 5).

On visual inspection of figures S1-4 (additional file 5) we found no evidence of differences in participant weight change or use of BCTs and recommendations by the GPN delivering the intervention.

\section{Comparison of BCT, dietary and physical activity recommendation use by weight loss of participants less or at least $5 \%$ from baseline at 6 months}

Thirty-nine (74\%) participants with audio recordings had 6-month weight data. Nineteen participants (49\%) had lost $\geq 5 \%$ of their baseline weight at 6 months and 59 consultations were recorded. The mean number and length of consultations per participant was 3.11 (SD 1.2) and 13.6 (SD 5.0) minutes respectively. In the 20 participants that lost $<5 \%$ of their weight (51\%), there were 62 recorded consultations. Mean number and length of consultations per participant was 3.15 (SD 1.0) and 12.6 (SD 4.8) minutes respectively. There were no significant differences in the mean length of consultations $(p=0.84)$ or total length of all audio-recorded consultations $(p=0.26)$ between the groups.

There were no significant differences between the two groups in the number of BCTs, dietary or physical activity recommendations used per consultation with the exception of social reward which was used significantly more in the group that lost $\geq 5 \%$ of their baseline weight. The BCTs and recommendations used in consultations and their comparisons are available in additional file 5, tables S2 and S3.

Table 2 Uses of BCTs, dietary and physical activity recommendations across all consultations

\begin{tabular}{lll}
\hline Number of & Mean (SD) & Range \\
\hline BCTs used per consultation per participant & $3.9(1.6)$ & $10.6(5.4)$ \\
BCTs used per participant over all of a participant's consultations & $3.3(2.7)$ & $3-9$ \\
Dietary recommendations used per consultation per participant & $8.4(6.6)$ & $0-12$ \\
Dietary recommendations used over all of a participant's consultations & $1.4(1.2)$ & $0-27$ \\
Physical activity recommendations used per consultation per participant & $3.6(3.5)$ & $0-5$ \\
Physical activity recommendations used over all of a participant's consultations & $0-11$ \\
\hline
\end{tabular}


A.

\begin{tabular}{llll} 
Usage in appointments (\%) & \multicolumn{1}{c}{ U } \\
0 & $20 \quad 30 \quad 40 \quad 50 \quad 60$
\end{tabular}

Feedback on outcome(s) of behaviour
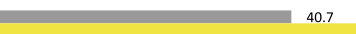

Information about health consequences
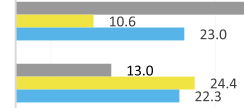

Framing/ reframing
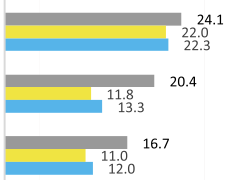$$
\begin{array}{ll}
4.9 & 20.4 \\
7.7 &
\end{array}
$$

B.

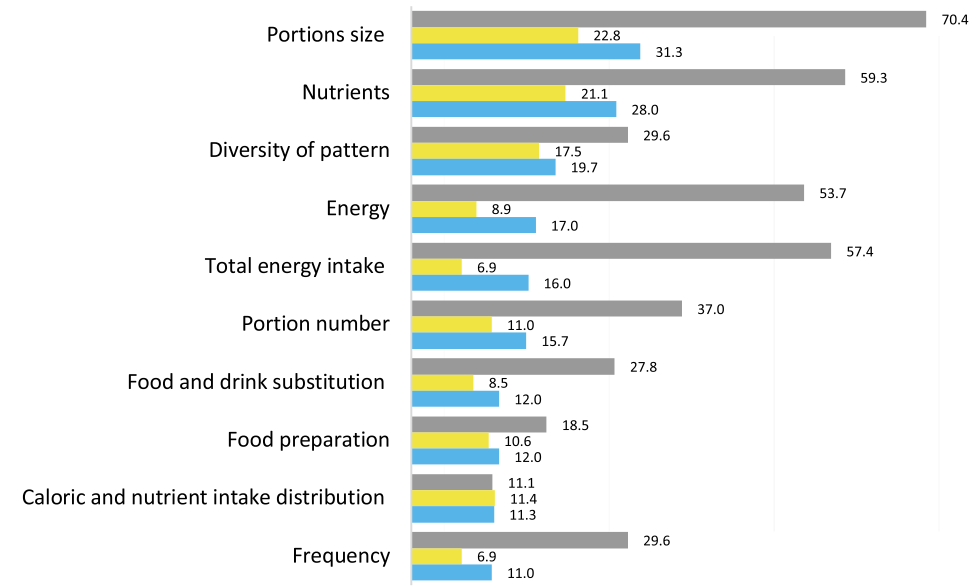

c.

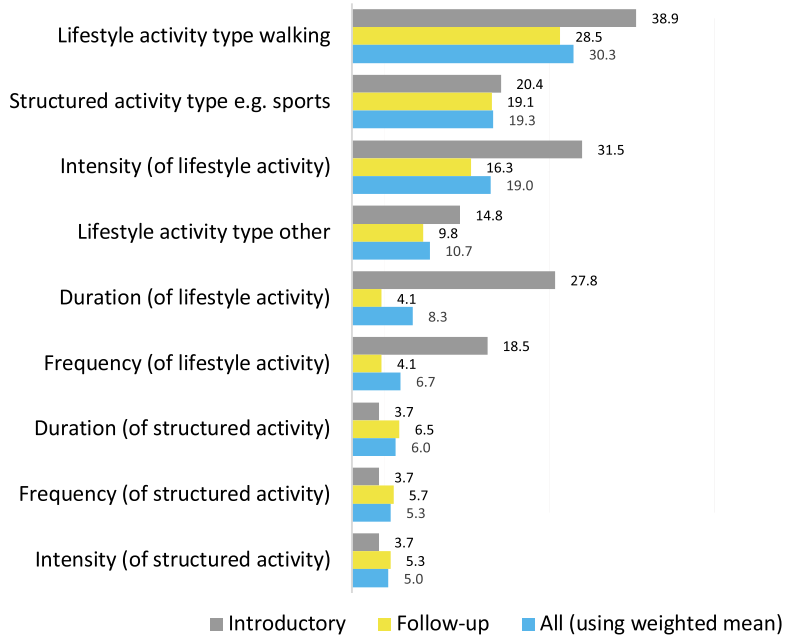

Fig. 1 The top ten most frequently used BCTs (a), dietary recommendations $(\mathbf{b})$ and physical activity recommendations (c) 
Table 3 Correlation coefficients ( $p$-values) between the total number of BCTs or recommendations used in introductory and all consultations of participants and percentage weight change at 3, 6 and 12 months

\begin{tabular}{|c|c|c|c|c|}
\hline & & $\begin{array}{l}\text { Total numbers } \\
\text { of BCTs coded }\end{array}$ & $\begin{array}{l}\text { Total number of dietary } \\
\text { recommendations coded }\end{array}$ & $\begin{array}{l}\text { Total number of physical } \\
\text { activity recommendations } \\
\text { coded }\end{array}$ \\
\hline \multirow[t]{2}{*}{ 3-month percentage weight change } & Introductory $(n=21)$ & $0.21(0.36)$ & $-0.07(0.76)$ & $0.10(0.65)$ \\
\hline & All consultations $(n=40)$ & ) $-0.21(0.19)$ & $-0.11(0.50)$ & $0.20(0.22)$ \\
\hline \multirow[t]{2}{*}{ 6-month percentage weight change } & Introductory $(n=20)$ & $0.17(0.49)$ & $-0.28(0.24)$ & $0.13(0.44)$ \\
\hline & All consultations $(n=39)$ & ) $-0.06(0.72)$ & $-0.14(0.38)$ & $0.23(0.16)$ \\
\hline \multirow[t]{2}{*}{ 12-month percentage weight change } & Introductory $(n=20)$ & $0.18(0.44)$ & $-0.22(0.35)$ & $0.34(0.14)$ \\
\hline & All consultations $(n=40)$ & $0.00(1.00)$ & $-0.03(0.87)$ & $0.29(0.07)$ \\
\hline
\end{tabular}

A negative correlation indicated that the number of techniques / recommendations was positively associated with weight loss

\section{Discussion}

Characterising the use of BCTs, dietary recommendations, and physical activity recommendations in this small group of GPN-led weight management consultations showed their use to be infrequent and variable. Despite consultations lasting longer $(13.8 \mathrm{~min})$ than typical primary care consultations $(9.2 \mathrm{~min})$ [21], few recognised BCTs, dietary or physical activity recommendations were used. Only $31 \%$ of BCTs were used and although $80 \%$ of the possible dietary recommendations were used, only $3 / 30$ recommendations were used per consultation. Only $1 / 10$ physical activity recommendation was used per consultation. GPNs used less than one $\mathrm{BCT}$ or recommendation per minute of consultation, and the number of BCTs or recommendations used were not significantly associated with weight loss.

This study has several strengths. Three different taxonomies were used to comprehensively characterise the relevant consultation components. The $\mathrm{BCT}$ taxonomy is well established, and the existing dietary taxonomy was adapted to improve characterisation. The novel physical activity taxonomy was developed based on an existing framework, and for the first time allowed coding of physical activity recommendations. GPNs were encouraged to carry out consultations as they had routinely done in their practice without the use of a prescriptive protocol, or additional training, allowing a more realistic characterisation of consultations in everyday clinical practice. Consultations were coded blind to outcome and inter-rater agreement levels were good. Although this is a specific and selected group of GPNs leading weight management consultations, this study indicates that what happens in "usual care" may or may not be grounded in guidelines. In the future, analysis of the content of usual care groups, potentially with the methods proposed here, and more standardised reporting, could help to identify effective components and improve reproducibility.

The study also has some limitations. About a third of consultations were recorded, which may reflect reluctance on the part of the GPN to record their consultations. There were no significant differences between weight change in the groups with and without audio recordings, but it is not possible to know whether the content of the recorded consultations are representative of the whole usual care group. Out of 11 GPNs, 8 GPNs submitted recordings and data were unavailable regarding the number or length of consultations participants without audio recordings received. There was no information on whether any additional consultations occurred outside of the recorded consultations, but we consider it unlikely that a significant number of additional consultations would exist, since this is neither routine care nor was advised in the trial. Overall, the small sample size limits the generalisability of our findings. The amount or type of training each GPN had on weight management was not recorded, but we found no evidence of differences in the components of the consultations or participants' weight loss between GPNs. Lack of formal analysis of the appropriateness and individualisation of recommendation use is also a limitation. Multiple testing might have led to increased probability for type I error, which limits our confidence in the few significant correlations identified. The observational design does not allow for casual inferences.

Previous research has found that primary care staff feel ill-equipped to help people with obesity, often reporting that they have inadequate training [22]. GPNs in this group, with previous training in weight management used only $46 \%$ of possible techniques/ recommendations on one occasion, and only $17 \%$ were used in more than $10 \%$ of consultations. Though it would not be expected for GPNs to use all BCTs and recommendations in one consultation, this low usage may reflect lack of 
awareness of effective weight-loss strategies. The literature suggests that increased and more adherent usage of BCTs is associated with greater weight loss [11], and the low and infrequent use of BCTs might explain the lack of association with weight loss shown here. Direct comparisons with other studies are difficult regarding dietary and physical activity recommendations as this is the first study using the taxonomies developed, but the lack of correlation between the frequency of dietary and physical activity recommendations given in consultations and weight loss may also result from low levels of use.

GPNs' use of techniques/ recommendations was confined to a small group of specific BCTs and recommendations. The most frequently used BCT was 'feedback on outcome of behaviour,' which has been linked to improved weight loss outcomes [23]. Reviews of weight management consultations commonly report 'self-monitoring of behaviour', used in almost $80 \%$ of introductory consultations, as one of the most effective BCTs to promote weight loss too $[24,25]$ and participants' weight loss, though modest, was better than other UK GPN led BMWCs $[8,26]$. 'Social reward' was the third most commonly used BCT and used significantly more in the group that lost $\geq 5 \%$ baseline weight at 6 months. NICE recommends healthcare professionals should 'praise successes' when offering weight management support [6] and it may be that as patients lost weight, GPNs congratulated patients. Additionally, it has been found that positive reinforcement of behaviour can be effective in other health behaviours, such as in increasing physical activity levels [27]. In terms of specific dietary recommendations, reference to 'energy' and 'total energy intake' were used in just over $15 \%$ of consultations characterised. Energy restriction is known to be a key component for weight loss [28, 29]. However, there was very limited discussion of specific energy deficits in the consultations and the lack of use in association with the BCT 'goal setting' could have limited weight loss here.

\section{Conclusions}

Specific dietary or physical activity recommendations and $\mathrm{BCTs}$ were used infrequently and variably in this small group of GPN-led weight management consultations. Further analysis in larger and representative samples is warranted. Future studies could usefully examine if additional training can increase the quality and effectiveness of consultations.

\section{Abbreviations}

$\mathrm{BCT}$ : Behaviour change technique; DROPLET: Doctor referral of overweight people to a low-energy treatment; GPN: General practice nurse; PHE: Public Health England; NICE: National Institute for Health and Care Excellence; RCT: Randomised control trial.

\section{Supplementary Information}

The online version contains supplementary material available at https://doi. org/10.1186/s12875-021-01403-1.

Additional file 1. BCTs, their descriptions and examples provided by BCTTV1 used by coders. This taxonomy has been taken directly from Michie et al's paper for reference.

Additional file 2. Dietary recommendations and their definitions used by coders. This has been adapted after taking directly from Stok et Al's paper.

Additional file 3. Coding rulebook.

Additional file 4. Physical activity recommendations and their definitions used by coders.

Additional file 5. Supplementary results.

\section{Acknowledgements}

We thank the participants for taking part in the trial, the GPNs who delivered the intervention, and the study co-investigators Paul Aveyard and Nerys Astbury.

\section{Authors' contributions}

SAJ conceived the study and obtained the funding. All authors designed the methods. HT conducted the data analysis supervised by DAK and EM and all authors contributed to the interpretation of the data. HT wrote the first draft of the manuscript and all authors critically revised and approved the final manuscript.

\section{Funding}

This study was funded by the National Institute for Health Research (NIHR) Oxford Biomedical Research Centre (grant number: IS-BRC-1215-20008). SAJ is an NIHR senior investigator and is also supported by NIHR Oxford Applied Research Collaboration. SAJ and DAK are supported by the National Institute for Health Research (NIHR) Oxford Biomedical Research Centre. EM is funded by a Wellcome Trust Clinical Doctoral Research Fellowship. The funder had no role in the design and conduct of the study; collection, management, analysis, and interpretation of the data; preparation, review, or approval of the manuscript; and decision to submit the manuscript for publication. The views expressed are those of the authors and not necessarily those of the NHS, the $\mathrm{NIHR}$, or the Department of Health and Social Care.

\section{Availability of data and materials}

The data are not publicly available due to them containing information that could compromise research participant privacy. Individual participant data that are deidentified and support the findings of this study are available from the chief investigator (SAJ) upon reasonable request. Requests for access to data should be sent to susan.jebb@phc.ox.ac.uk. All proposals requesting data access will need to complete a data request form with details of the research question and analysis plan. All proposals will require the approval of the investigator team before any data are released.

\section{Declarations}

\section{Ethics approval and consent to participate}

This trial was approved by South Central Oxford B NHS Research Ethics Service Committee (NHS REC No 15/SC/0337). Participants provided written informed consent for their consultations to be recorded.

\section{Consent for publication}

Not applicable.

\section{Competing interests}

SAJ was chief investigator of the DROPLET trial which was part-funded by the Cambridge Weight Plan. DAK and SAJ report being investigators in a NIHRfunded trial where the weight loss intervention is provided by Nestle Health Sciences. SAJ spoke at a meeting on digital health hosted by Oviva for which expenses and an honorarium were paid to the University of Oxford. The other authors have declared no competing interests. 


\section{Author details}

${ }^{1}$ Nuffield Department of Primary Care Health Sciences, University, of Oxford, Radcliffe Observatory Quarter, Woodstock Road, Oxford OX2 6GG, UK. ${ }^{2} \mathrm{NIHR}$ Oxford Biomedical Research Centre, Oxford, UK.

Received: 23 September 2020 Accepted: 24 February 2021 Published online: 07 April 2021

\section{References}

1. Health Effects of Overweight and Obesity in 195 Countries over 25 Years. New England Journal of Medicine. 2017;377(1):13-27. https://doi.org/10. 1056/NEJMoa1614362.

2. Dee A, Kearns K, O'Neill C, Sharp L, Staines A, O'Dwyer V, et al. The direct and indirect costs of both overweight and obesity: a systematic review. BMC Research Notes. 2014;7(1):242. https://doi.org/10.1186/ 1756-0500-7-242.

3. Tuomilehto J, Lindström J, Eriksson JG, Valle TT, Hämäläinen H, IlanneParikka P, et al. Prevention of Type 2 Diabetes Mellitus by Changes in Lifestyle among Subjects with Impaired Glucose Tolerance. N Engl J Med. 2001;344(18):1343-50. https://doi.org/10.1056/nejm200105033441801.

4. Brown JD, Buscemi J, Milsom V, Malcolm R, O'Neil PM. Effects on cardiovascular risk factors of weight losses limited to 5-10. Transl Behav Med. 2016;6(3):339-46. https://doi.org/10.1007/s13142-015-0353-9.

5. Kritchevsky SB, Beavers KM, Miller ME, Shea MK, Houston DK, Kitzman DW, et al. Intentional Weight Loss and All-Cause Mortality: A Meta-Analysis of Randomized Clinical Trials. PLoS ONE. 2015;10(3):e0121993. https://doi. org/10.1371/journal.pone.0121993.

6. Centre for Public Health Excellence at Nice. Obesity: The Prevention, Identification, Assessment and Management of Overweight and Obesity in Adults and Children. National Institute for Health and Clinical Excellence (UK); 2006. https://www.nice.org.uk/guidance/cg189.

7. Carels RA, Young KM, Coit C, Clayton AM, Spencer A, Hobbs M. Can following the caloric restriction recommendations from the Dietary Guidelines for Americans help individuals lose weight? Eat Behav. 2008;9(3):328-35. https://doi.org/10.1016/j.eatbeh.2007.12.003.

8. Jolly K, Lewis A, Beach J, Denley J, Adab P, Deeks JJ, et al. Comparison of range of commercial or primary care led weight reduction programmes with minimal intervention control for weight loss in obesity: Lighten Up randomised controlled trial. BMJ. 2011;343:d6500. https://doi.org/10.1136/bmj.d6500.

9. Booth HP, Prevost TA, Wright AJ, Gulliford MC. Effectiveness of behavioural weight loss interventions delivered in a primary care setting: a systematic review and meta-analysis. Fam Pract. 2014;31(6):643-53. https://doi.org/ 10.1093/fampra/cmu064.

10. Hoffmann TC, Glasziou PP, Boutron I, Milne R, Perera R, Moher D, et al. Better reporting of interventions: template for intervention description and replication (TIDieR) checklist and guide. BMJ : British Medical Journal. 2014;348:g1687. https://doi.org/10.1136/bmj.g1687.

11. Hankonen N, Sutton S, Prevost AT, Simmons RK, Griffin SJ, Kinmonth AL, et al. Which behavior change techniques are associated with changes in physical activity, diet and body mass index in people with recently diagnosed diabetes? Ann Behav Med. 2015;49(1):7-17. https://doi.org/10. 1007/s12160-014-9624-9.

12. Astbury NM, Aveyard P, Nickless A, Hood K, Corfield K, Lowe R, et al. Doctor Referral of Overweight People to Low Energy total diet replacement Treatment (DROPLET): pragmatic randomised controlled trial. BMJ. 2018;362:k3760. https://doi.org/10.1136/bmj.k3760.

13. Michie S, Richardson M, Johnston M, Abraham C, Francis J, Hardeman W, et al. The Behavior Change Technique Taxonomy (v1) of 93 Hierarchically Clustered Techniques: Building an International Consensus for the Reporting of Behavior Change Interventions. Ann Behav Med. 2013;46(1):81-95. https://doi.org/10.1007/s12160-013-9486-6.

14. Marijn Stok F, Renner B, Allan J, Boeing H, Ensenauer R, Issanchou S, et al. Dietary Behavior: An Interdisciplinary Conceptual Analysis and Taxonomy. Front Psychol. 2018;9:1689. https://doi.org/10.3389/fpsyg.2018.01689.

15. Behaviour change: individual approaches. https://www.nice.org.uk/guida nce/ph49/chapter/6-Glossary. Accessed 20 Nov 2019.

16. Riebe D, Ehrman JK, Liguori G, Magal M. ACSM's guidelines for exercise testing and prescription. Tenth edition ed. Philadelphia: Wolters Kluwer; 2017.
17. Health matters: getting every adult active every day. https://www.gov. uk/government/publications/health-matters-getting-every-adultactive-every-day/health-matters-getting-every-adult-active-every-day. Accessed 27 Feb 2020.

18. Biswas A, Oh PI, Faulkner GE, Bajaj RR, Silver MA, Mitchell MS, et al. Sedentary time and its association with risk for disease incidence, mortality, and hospitalization in adults: a systematic review and meta-analysis. Ann Intern Med. 2015;162(2):123-32. https://doi.org/10.7326/m14-1651.

19. BCT Taxonomy: Starter Pack For Trainees. http://www.bct-taxonomy.com/ pdf/StarterPack.pdf. Accessed 27 Feb 2020.

20. Prochaska JO, DiClemente CC. Stages and processes of self-change of smoking: toward an integrative model of change. J Consult Clin Psychol. 1983;51(3):390-5. https://doi.org/10.1037//0022-006x.51.3.390.

21. Hobbs FDR, Bankhead C, MukhtarT, Stevens S, Perera-Salazar R, Holt T, et al. Clinical workload in UK primary care: a retrospective analysis of 100 million consultations in England, 2007-14. The Lancet. 2016;387(10035):2323-30. https://doi.org/10.1016/S0140-6736(16) 00620-6.

22. Bucher Della Torre S, Courvoisier DS, Saldarriaga A, Martin XE, FarpourLambert NJ. Knowledge, attitudes, representations and declared practices of nurses and physicians about obesity in a university hospital: training is essential. Clin Obes. 2018;8(2):122-30. https://doi.org/10.1111/ cob.12238.

23. O'Neil PM, Brown JD. Weighing the Evidence: Benefits of Regular Weight Monitoring for Weight Control1. Journal of Nutrition Education and Behavior. 2005;37(6):319-22. https://doi.org/10.1016/S1499-4046(06) 60163-2.

24. Olander EK, Fletcher H, Williams S, Atkinson L, Turner A, French DP. What are the most effective techniques in changing obese individuals' physical activity self-efficacy and behaviour: a systematic review and metaanalysis. Int J Behav Nutr Phys Act. 2013;10:29. https://doi.org/10.1186/ 1479-5868-10-29.

25. Michie S, Abraham C, Whittington C, McAteer J, Gupta S. Effective techniques in healthy eating and physical activity interventions: a metaregression. Health Psychol. 2009;28(6):690-701. https://doi.org/10.1037/ a0016136.

26. Counterweight Project T. Evaluation of the Counterweight Programme for obesity management in primary care: a starting point for continuous improvement. Br J Gen Pract. 2008;58(553):548-54. https://doi.org/10. 3399/bjgp08X319710.

27. Geerling R, Browne JL, Holmes-Truscott E, Furler J, Speight J, Mosely K. Positive reinforcement by general practitioners is associated with greater physical activity in adults with type 2 diabetes. BMJ Open Diabetes Research \& Care. 2019;7(1):e000701. https://doi.org/10.1136/ bmjdrc-2019-000701.

28. Samdal GB, Eide GE, Barth T, Williams G, Meland E. Effective behaviour change techniques for physical activity and healthy eating in overweight and obese adults; systematic review and meta-regression analyses. Int J Behav Nutr Phys Act. 2017;14(1):42. https://doi.org/10.1186/ s12966-017-0494-y.

29. Hartmann-Boyce J, Johns DJ, Jebb SA, Summerbell C, Aveyard P, Behavioural Weight Management Review G. Behavioural weight management programmes for adults assessed by trials conducted in everyday contexts: systematic review and meta-analysis. Obes Rev. 2014;15(11):920-32. https://doi.org/10.1111/obr.12220.

\section{Publisher's Note}

Springer Nature remains neutral with regard to jurisdictional claims in published maps and institutional affiliations. 